\title{
1,8-dihydroxy-3-acetyl-6-methyl-9,10 anthraquinone exhibits a potent radiosensitizing effect with induced oncosis in human nasopharyngeal carcinoma cells
}

\author{
HUAXIN HOU ${ }^{1}$, DANRONG $\mathrm{LI}^{2}$, WEI JIANG ${ }^{1}$, YAN LIANG ${ }^{1}$, DONGLIAN CHEN ${ }^{1}$ and YUANYUAN MO ${ }^{1}$ \\ ${ }^{1}$ Department of Pharmaceutical Analysis, College of Pharmacy, Guangxi Medical University; ${ }^{2}$ Department of Cellular Biology, \\ The Medical Scientific Research Centre of Guangxi Medical University, Nanning, Guangxi 530021, P.R. China
}

Received September 18, 2013; Accepted April 14, 2014

DOI: $10.3892 / \mathrm{mmr} .2014 .2307$

\begin{abstract}
. 1,8-dihydroxy-3-acetyl-6-methyl-9,10 anthraquinone (DAMA) was synthesized from emodin. In the present study, the activity and the oncosis-like mechanism of DAMA-enhanced nasopharyngeal carcinoma (NPC) cell sensitivity to ionizing radiation was examined. The results demonstrated that DAMA has a 1.46-fold radiosensitisation activity for nasopharyngeal carcinoma CNE 1 cells with a non-cytotoxic concentration of $10 \mu \mathrm{g} / \mathrm{ml}$ DAMA combined with 2 Gy. Following treatment of DAMA combined with radiation, CNE-1 cells revealed severe cytoplasmic swelling and vacuolization, swollen mitochondria and dilation of the nuclei without chromatin condensation, yielding a typical morphology of oncosis. Oncosis-related gene expression of ATP synthase F0 subunit 6, chromatin modifying protein 6 and cyclophilin D mRNA increased significantly in the $8 \mathrm{~Gy}$ radiation group and the 2 Gy radiation combined with DAMA group. A significant decrease of ATP synthase protein 8 mRNA was observed and the levels of intracellular ATP were also reduced. In addition, the levels of intracellular $\mathrm{Ca}^{2+}$ were increased. In conclusion, DAMA is a potent radiation sensitizer in nasopharyngeal carcinoma cells and mediates its radiosensitisation via oncosis.
\end{abstract}

\section{Introduction}

Nasopharyngeal carcinoma (NPC) is common in southern regions of China. Radiotherapy is the main therapeutic strategy for the treatment of patients with NPC, however $>35 \%$ of patients relapse following radiotherapy. Resistance to radiation remains a major obstacle for the successful treatment of

Correspondence to: Professor Danrong Li, Department of Cellular Biology, The Medical Scientific Research Centre of Guangxi Medical University, 22 Shuangyong Road, Nanning, Guangxi 530021, P.R. China E-mail: danrongli@163.com

Key words: radiosensitizer, nasopharyngeal carcinoma, 1,8-dihydroxy-3-acetyl-6-methyl-9,10 anthraquinone, oncosis
NPC (1). Therefore, the development of a novel radiosensitizer that enhances NPC sensitivity to ionizing radiation is urgently required. An increasing number of studies have demonstrated that the bioreductive agent emodin (1,3,8-trihydroxy-6-methylanthraquinone) is able to reverse multidrug resistance or enhance the cytotoxicity of chemotherapeutic drugs. In our previous study, it was revealed that emodin possesses radiosensitizing effects in NPC cells in vitro and in vivo $(2,3)$. In order to obtain a higher number of possible therapeutic agents, 1,8-dihydroxy-3-acetyl-6-methyl-9,10 anthraquinone (DAMA) was synthesized from emodin (4). In the present study it was investigated whether the DAMA compound was able to increase the radiosensitivity of the CNE-1 NPC cell line in vitro. Confocal microscopy and electron microscopy were employed to characterize the mechanism of action associated with mitochondrial dysfunction and the level of intercellular $\mathrm{Ca}^{2+}$. In particular, the study aimed to determine whether DAMA-treated cancer cells exhibit a profile consistent with an oncosis-like (non-apoptotic) mechanism.

\section{Materials and methods}

Cell culture. Human nasopharyngeal carcinoma CNE-1 cells were obtained from the Shanghai Institutes for Biological Sciences of the Chinese Academy of Sciences (Shanghai, China). The CNE-1 human NPC cell line, was grown in culture medium RPMI-1640 (Gibco-BRL, Grand Island, NY, USA) containing $10 \%(\mathrm{v} / \mathrm{v})$ heat-inactivated newborn bovine serum (NBS; Gibco-BRL), $100 \mu \mathrm{g} / \mathrm{ml}$ streptomycin (Lukang Pharmaceutical, Shandong, China) and $100 \mathrm{IU} / \mathrm{ml}$ penicillin (Lukang Pharmaceutical) at $37^{\circ} \mathrm{C}$ in a humidified atmosphere with $5 \% \mathrm{CO}_{2}$. All of the cultures were examined routinely and found to be free of contamination by mycoplasma or fungi.

Reagents and chemicals. DAMA (98\% purity) was synthesized and the chemical properties were consistent with the literature reported (4). It was fully dissolved as a stock solution in $100 \%$ ethyl alcohol at $1 \mathrm{~g} / \mathrm{l}$. For the cell treatments, the drugs were further diluted in culture medium to the required concentrations with a final ethyl alcohol concentration of $<1.6 \%$. MTT was obtained from Sigma (St. Louis, MO, USA) and dimethylsulfoxide (DMSO) was obtained from Bodi Chemical (Tianjin, 
Table I. Primer sequences of oncosis-related genes.

\begin{tabular}{lllr}
\hline Gene & Strand & \multicolumn{1}{c}{ Primer seq (5'-3') } & $\begin{array}{r}\text { Product } \\
\text { size }(\mathrm{bp})\end{array}$ \\
\hline ATP6 & Sense & \multicolumn{1}{c}{ GTGATTATAGGCTTTCGCTCT } \\
& Antisense & CAGTAATGTTAGCGGTTAGGC & 160 \\
ATP8 & Sense & ACTCCTTACACTATTCTCATCAC & 147 \\
$\beta$-actin & Antisense & GGCAATGAATGAAGCGAACAG & 247 \\
& Sense & AACTCCATCATGAAGTGTGA & 185 \\
CYPD & Antisense & ACTCCTGCTTGCTGATCCAC & 149 \\
CHMP6 & Sense & GTTATTGAGACAGCAGATAGAG & \\
& Antisense & AATCCTTGCCATCCTTGAG & \\
\end{tabular}

ATP6, ATP synthase F0 subunit 6; ATP8, ATP synthase protein 8; CYPD, cyclophilin D; CHMP6, chromatin modifying protein 6.

China). TRIzol reagent was obtained from Invitrogen Life Technologies (Carlsbad, CA, USA). RevertAid first strand cDNA synthesis kit was purchased from MBI Fermentas (Hanover, $\mathrm{MD}, \mathrm{USA})$. All of the polymerase chain reaction (PCR) reagents were purchased from Takara Biotech (Dalian, China).

MTT assay. The non cytotoxic dose (growth rate $>90 \%$ ) of DAMA for CNE- 1 cells was $10 \mu \mathrm{g} / \mathrm{ml}$ and thus the non cytotoxic dose of DAMA was selected as the final experimental concentration. The MTT assay was performed as described previously with minor modifications (11). Briefly, the CNE-1 cells were harvested with trypsin and resuspended to a final density of $1 \times 10^{5}$ cells $/ \mathrm{ml}$. Aliquots of $100 \mu \mathrm{l}$ from each cell suspension were distributed evenly into Costar 96-well cell culture plates (Gibco-BRL). Following incubation of the cells for $24 \mathrm{~h}$, the designated wells were treated with different concentrations of DAMA. Following incubation for $48 \mathrm{~h}$, $20 \mu \mathrm{l} \mathrm{MTT}$ solution $(5 \mathrm{mg} / \mathrm{ml})$ was added into each well and incubated at $37^{\circ} \mathrm{C}$ in a $5 \% \mathrm{CO}_{2}$ atmosphere for $4 \mathrm{~h}$. Next, the solution was removed from the wells and the formazan crystals were solubilized in $200 \mu \mathrm{l}$ DMSO in every well. The reduction of MTT was quantified by the absorbance at a wavelength of 490 nm using a Multiskan MK3 (Thermo Fisher Scientific, Waltham, MA, USA). Three wells were measured in each group. The percentage of inhibition was calculated as follows: $\%$ inhibition $=[1-($ mean $\mathrm{A}$ of sample/mean $\mathrm{A}$ of control $)] \mathrm{x} 100$.

Clonogenic cell survival assay. The CNE-1 cells were trypsinized and counted. The appropriate number of cells were plated in $60-\mathrm{mm}$ dishes and allowed to attach for $24 \mathrm{~h}$. Following treatment with $5 \mathrm{mg} / \mathrm{l}$ and $10 \mathrm{mg} / \mathrm{l}$ of DAMA for $24 \mathrm{~h}$, the cells were irradiated $(2,4,6,8 \mathrm{~Gy})$ and incubated for 8-10 days. The colonies were stained with crystal violet (Sigma Chemical Co., St. Louis, MO, USA), and colonies of $\geq 50$ cells were counted. Clonogenic fractions of irradiated cells were normalised to the plating efficiency (PE) of the unirradiated controls. The PE was calculated as follows: $\mathrm{PE}=$ (colonies counted/cells inoculated) $\mathrm{x} 100$. The number of colonies that arise following treatment of cells, expressed in terms of PE, was termed the surviving fraction (SF). The surviving fraction was calculated as follows: no. of colonies formed following treatment/(no. of cells seeded $x$ PE).

Quantitative (q)PCR. Total RNA was extracted by TRIzol (Invitrogen Life Technologies) and reverse transcribed by Superscript III (Invitrogen Life Technologies). The qPCR experiments were performed using the Roche SYBR Green PCR Master mix with the ABI 7500 Real-Time PCR system (Applied Biosystems, Foster City, CA, USA). All of the experimental procedures were performed according to the manufacturer's instructions. qPCR primer sequences are comprehensively listed in Table I. The remaining procedures were performed identically to mRNA quantification.

Mitochondrial membrane potential assays. Rhodamine 123 [2-(6-Amino-3-imino-3H-xanthen-9-yl) benzoic acid methyl ester, hydrochloride] is also used in biochemistry to inhibit mitochondrial function. It appears to bind to the mitochondrial membranes and inhibit transport processes. Mitochondrial energization induces quenching of Rhodamine 123 fluorescence and the rate of fluorescence decay is proportional to the mitochondrial membrane potential. Each group of cells were harvested and centrifuged at $400 \mathrm{x} \mathrm{g}$ for $5 \mathrm{~min}$, and the cell pellet was resuspended in $0.5 \mathrm{ml} \mathrm{Rh} 123$ solution $(10 \mu \mathrm{g} / \mathrm{ml})$ for $10 \mathrm{~min}$. The cells were then washed and resuspended in phosphate-buffered saline for confocal microscope analysis using a Nikon A1 confocal microscope (Nikon, Tokyo, Japan).

Determination of intracellular ATP concentration. The level of available intracellular ATP in CNE-1 cell lines was measured as an indirect parameter of the activity of ATPase. A commercially available ATP determination kit (Beyotime Institute of Biotechnology, Shanghai, China) based on luciferase activity was used according to the manufacturer's instructions.

Determination of intracellular $\mathrm{Ca}^{2+}$ concentration by confocal laser fluorescent microscopy. Fluo 3 AM is a long wavelength calcium probe that is practically non-fluorescent in 


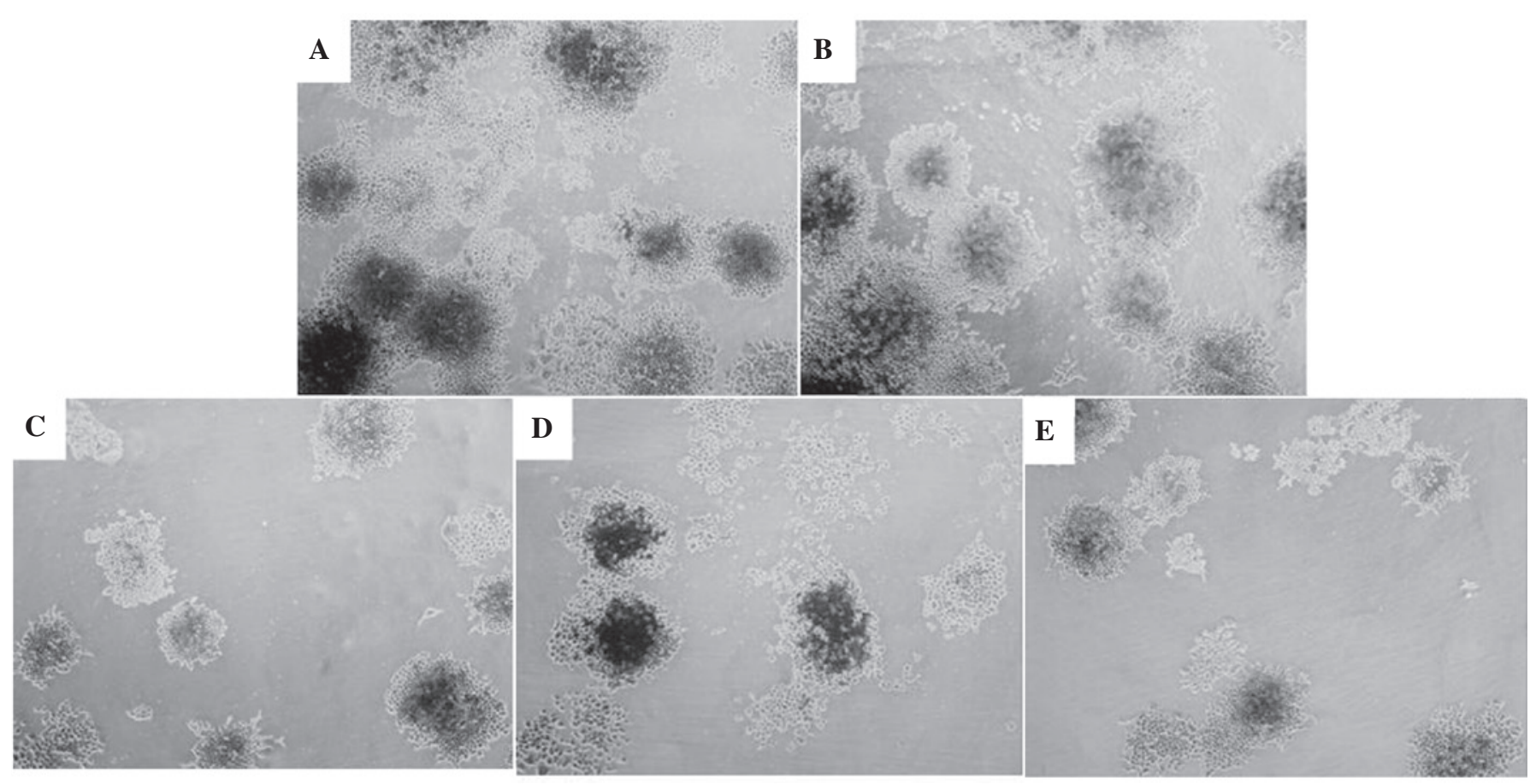

Figure 1. Effect of emodin on colony formation in CNE-1 cells. Changes in cell colony morphology were visualized using an inverted microscope (magnification, x40). The cells were fixed with $4 \%$ paraformaldehyde and stained with Giemsa. (A) Control; (B) 2 Gy; (C) 8 Gy; (D) $10 \mu \mathrm{g} / \mathrm{ml}$ DAMA; (E) DAMA $(10 \mu \mathrm{g} / \mathrm{ml})+2$ Gy. DAMA, 1,8-dihydroxy-3-acetyl-6-methyl-9,10 anthraquinone.

its free ligand form, but its fluorescence increases 50-100 times when it forms complexes with calcium. Therefore, it has been widely used with confocal laser fluorescent microscopy for the determination of the calcium loaded into cells by incubation. Each group of cells were harvested and centrifuged at $400 \mathrm{x} \mathrm{g}$ for $5 \mathrm{~min}$. Aliquots of the cell suspension were incubated with $10 \mathrm{mM}$ fura-3AM with pluronic (2\%) in medium. Following loading with fura-3AM at $37^{\circ} \mathrm{C}$ for $30 \mathrm{~min}$, the cells were washed twice for $3 \mathrm{~min}$ each time, then centrifuged at $400 \mathrm{x} \mathrm{g}$ for $5 \mathrm{~min}$ to remove extracellular fluorophores and resuspended in $1.2 \mathrm{ml}$ of the same medium without albumin for confocal microscope analysis.

Statistical analysis. All experiments were performed at least three times. Statistical analysis was performed using one-way analysis of variance to compare the effect among the control and treated cells. $\mathrm{P}<0.05$ was considered to indicate a statistically significant difference.

\section{Results}

Modulation of radiation resistance by DAMA. The colony numbers and the surviving fraction of CNE-1 cells treated with $10 \mu \mathrm{g} / \mathrm{ml}$ DAMA combined with radiation are demonstrated in Table II and Fig. 1, respectively. The survival curve and the sensitization enhancement effect of DAMA are demonstrated in Fig. 2. There were evident effects on the radiosensitivity of CNE-1 cells exposed to DAMA at non-cytotoxic concentrations $(10 \mu \mathrm{g} / \mathrm{ml})$. There was a dose modifying factor (DMF) of 1.46 .

Cell ultrastructure. Electron microscopy (Hitachi 7650 electron microscope; Hitachi, Tokyo, Japan) confirmed that
Table II. Parameters of cell survival curve.

\begin{tabular}{lcc}
\hline Group & $\mathrm{D}_{0}$ & $\mathrm{DMF}$ \\
\hline Control group & 5.63 & \\
DAMA & 3.86 & 1.46 \\
\hline
\end{tabular}

DAMA, 1,8-dihydroxy-3-acetyl-6-methyl-9,10 anthraquinone; DMF, dose modification factors.

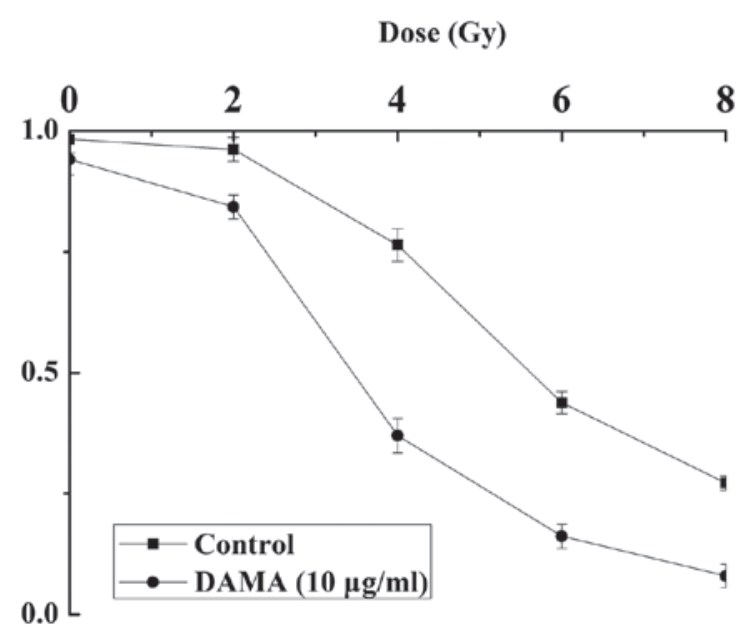

Figure 2. Survival curve of CNE-1 cells. DAMA, 1,8-dihydroxy-3-acetyl-6methyl-9,10 anthraquinone.

the control CNE-1 cells had an intact cellular morphology with ultrastructurally normal nuclei and organelles (Fig. 3A). By contrast, the CNE-1 cells treated with $10 \mu \mathrm{g} / \mathrm{ml}$ DAMA 

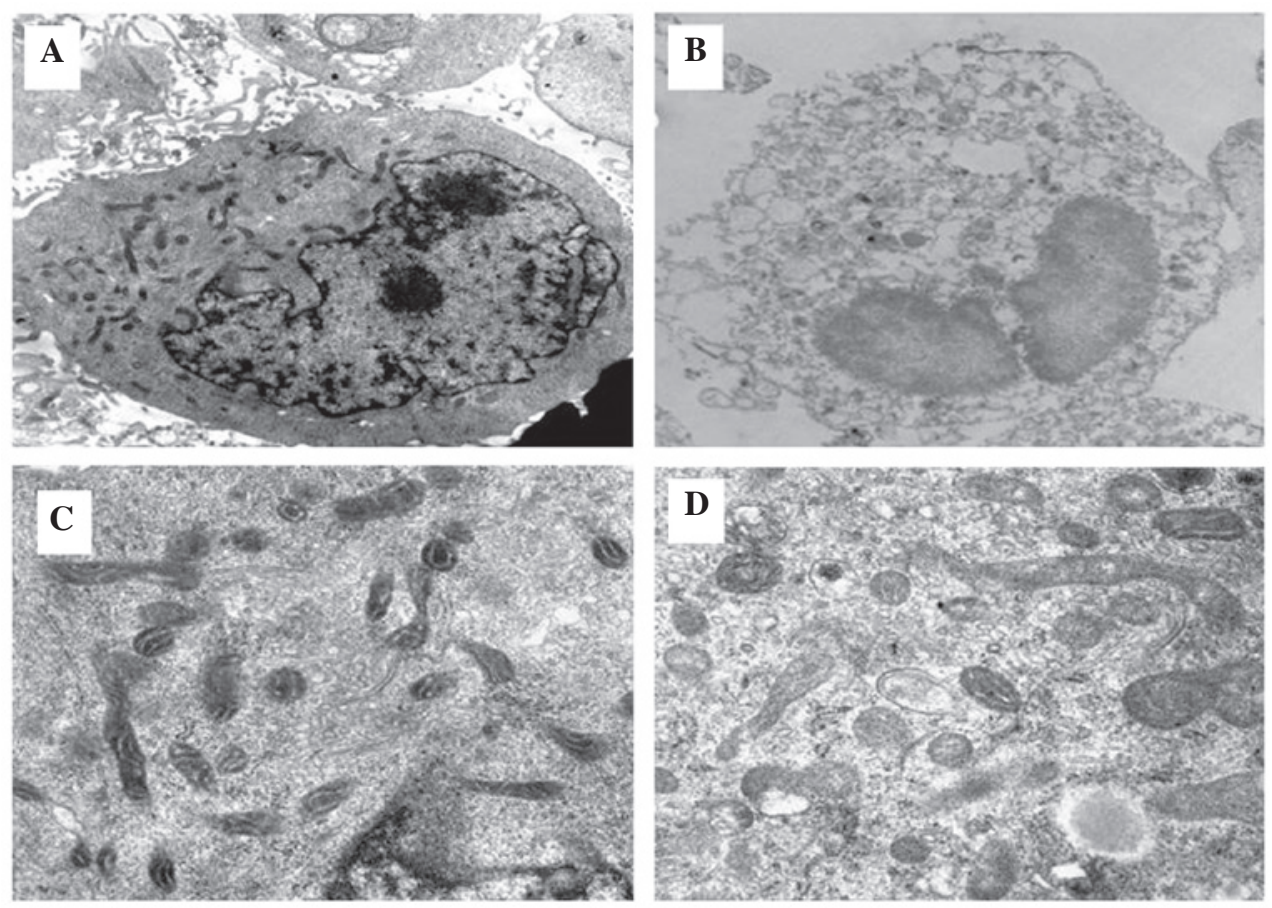

Figure 3. DAMA-mediated cell death features resemble ultrastructural features of oncosis-like cell death. (A) Control cells demonstrating intact ultrastructurally normal nuclei and cellular organelles and non-cytoplasmic vacuolation. (B) Numerous large and small cytoplasmic vacuoles, dilation of nuclei, ruptured plasma membrane late in the process of $10 \mu \mathrm{g} / \mathrm{ml}$ DAMA combination with radiation-treated cell death. (C) The mitochondria in the control group cells. (D) Severely swollen mitochondria in the DAMA combined with radiation group. DAMA, 1,8-dihydroxy-3-acetyl-6-methyl-9,10 anthraquinone.

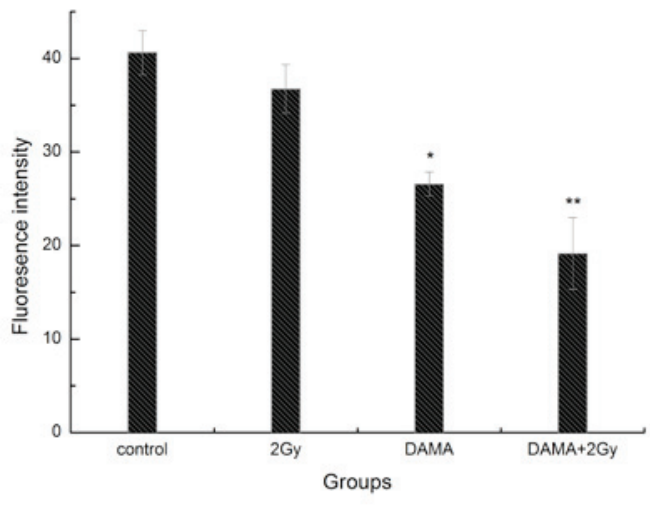

Figure 4. The fluorescence intensity of the membrane potential in CNE-1 cells. (A) Control group; (B) 2 Gy radiation group; (C) $10 \mu \mathrm{g} / \mathrm{ml}$ DAMA group; (D) $10 \mu \mathrm{g} / \mathrm{ml}$ DAMA combined with 2 Gy radiation group. DAMA, 1,8-dihydroxy-3-acetyl-6-methyl-9,10 anthraquinone. ${ }^{*} \mathrm{P}<0.05,{ }^{* *} \mathrm{P}<0.01$, compared with the control.

and radiation for $48 \mathrm{~h}$ exhibited cytoplasmic swelling with a marked disruption of cytoarchitecture, including numerous small and large cytoplasmic vacuoles as well as swollen and internally disorganized mitochondria. The most prominent nuclear changes were dilation of the nuclei, irregular clumping of chromatin and the appearance of cleared chromatin-free nuclear domains (Fig. 3B-D).

Alteration of the mitochondrial transmembrane potential. The fluorescence intensity was $40.63 \pm 2.36$ in the control group. In

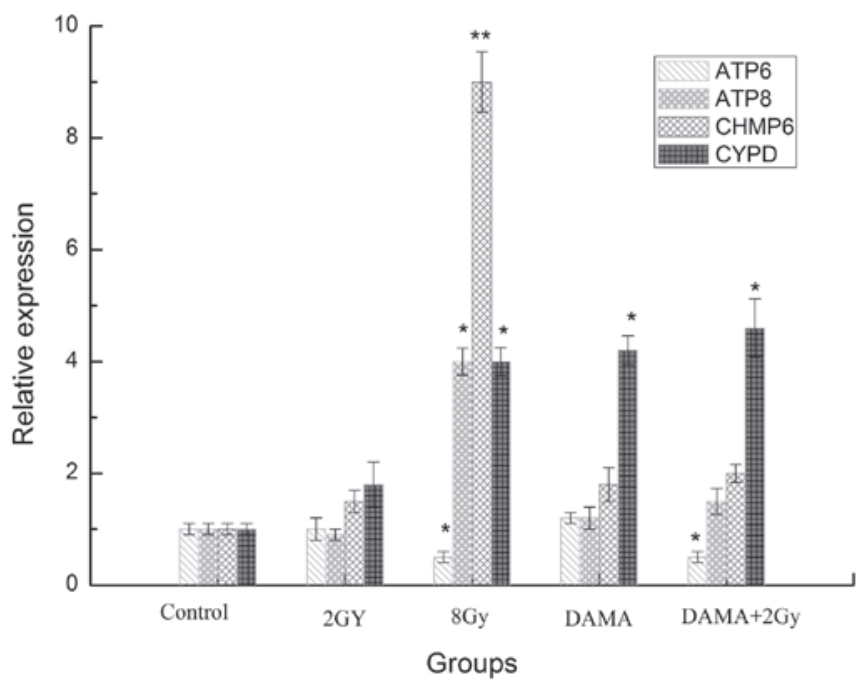

Figure 5. ATP6, ATP8, CHMP6, CYPD mRNA expression of CNE-1 cells treated by DAMA. DAMA, 1,8-dihydroxy-3-acetyl-6-methyl-9,10 anthraquinone; CHMP6, chromatin modifying protein 6; CYPD, cyclophilin D; ATP6, ATP synthase F0 subunit 6; ATP8, ATP synthase protein 8 . "P<0.05, ${ }^{* *} \mathrm{P}<0.01$, compared with the control.

the 2 Gy radiation group and DAMA group, the values were $36.73 \pm 2.62$ and $26.56 \pm 1.29$, respectively. Their mitochondrial transmembrane potentials were significantly decreased $(\mathrm{P}<0.05)$ compared with that of the control group. Notably, the fluorescence intensity of the radiosensitization group D was $19.14 \pm 3.84$, thus the mitochondrial transmembrane potential was evidently decreased. The difference compared with the control group was statistically significant $(\mathrm{P}<0.01 ;$ Fig. 4). 
Table III. Fluorescence intensity of $\mathrm{Ca}^{2+}$ in $\mathrm{CNE}-1$ cells.

\begin{tabular}{lcc}
\hline Group & $\begin{array}{c}\text { Relative ATP level } \\
(\%)\end{array}$ & $\begin{array}{c}\text { Ca }^{2+} \text { fluorescence intensity } \\
(\text { mean } \pm \text { SD) }\end{array}$ \\
\hline Control & 100.0 & $1164.17 \pm 68.69$ \\
DAMA & $75.3 \pm 10.3$ & $1391.83 \pm 33.35^{\mathrm{a}}$ \\
2 Gy & $55.6 \pm 6.4$ & $1406.00 \pm 48.02^{\mathrm{a}}$ \\
DAMA + 2 Gy & $45.7 \pm 5.3$ & $1940.08 \pm 55.74^{\mathrm{b}}$ \\
\hline
\end{tabular}

${ }^{\mathrm{a}} \mathrm{P}<0.05,{ }^{\text {b }}<0.01$ compared with control. DAMA, 1,8-dihydroxy-3-acetyl-6-methyl-9,10 anthraquinone.

mRNA expression of oncosis-related genes in CNE-1 cells treated by DAMA. As revealed in Fig. 5, the mRNA expression of the oncosis-related genes, ATP synthase protein 8 (ATP8), chromatin modifying protein 6 (CHMP6) and cyclophilin D (CYPD), increased significantly in the 8 Gy radiation group and 2 Gy radiation combined with DAMA group. A significant decrease in the ATP synthase F0 subunit 6 (ATP6) mRNA expression was observed.

Intracellular concentration of ATP and $\mathrm{Ca}^{2+}$ in each group. The fluorescence intensity was $1164.17 \pm 68.69$ in the control group following Fluo-3 AM staining. The fluorescence intensity was $1391.83 \pm 33.35$ and $1406.0 \pm 48.02$ in the 2 Gy group and $10 \mu \mathrm{g} / \mathrm{ml}$ DAMA group, respectively, which was significantly different compared with the control group $(\mathrm{P}<0.05)$. The fluorescence intensity was $1940.08 \pm 55.74$ in the 2 Gy combined with $10 \mu \mathrm{g} / \mathrm{ml}$ DAMA group, which was significantly increased compared with the 2 Gy group and $10 \mu \mathrm{g} / \mathrm{ml}$ DAMA group $(\mathrm{P}<0.05)$ as well as the control group $(\mathrm{P}<0.01)$. The results demonstrated that there was evident $\mathrm{Ca}^{2+}$ overloading in the cells. Following treatment with DAMA, or DAMA combined with 2 Gy, ATP levels decreased to 75.3 and $45.7 \%$, respectively (Table III). It has been demonstrated that the intracellular ATP level is an important factor in deciding the form of cell death when cells were exposed to a lethal stimulation (5). Lieberthal et al (6) demonstrated that the proximal tubule cells subjected to severe ATP depletion die by necrosis, whereas moderate ATP depletion results in apoptosis. Our results suggested that depletion of ATP may convert the cellular morphology from apoptosis- to oncosis-typical characteristics, suggesting that intracellular ATP levels regulate the mode of cell death.

\section{Discussion}

The cell death pathway is divided into apoptosis and oncosis according to the American Society of Toxicology Pathology (7). Oncosis is a pathway of cell death that is different from apoptosis, and which has become the focus of a number of morphological studies in recent years (8-10). In the present study, it was identified that DAMA significantly enhanced NPC cell sensitivity to radiation therapy. The ultrastructure of the control cells was observed by transmission electron microscopy, and it was noted that the mitochondria were circular or ovoid, the mitochondria cristae were clearly visible and there was no swelling or cavities. Following treat- ment with radiotherapy combined with DAMA, the NPC cells exhibited mitochondrial cytoplasmic swelling with a marked disruption of cytoarchitecture, including numerous small and large cytoplasmic vacuoles as well as swollen and internally disorganized mitochondria. Therefore, it appears that induced mitochondria oncosis may be an important radiosensitization mechanism of DAMA.

As is well established, mitochondria have an important role in the cell death pathway $(11,12)$. Mitochondria are the main cellular organelle that produce ATP and store energy. Previous studies suggest that apoptosis and oncosis may be triggered by the same types of stimulation and depend on the stimulus intensity and duration time. When the stimulus intensity is weak, the activity duration is relatively short and apoptosis is prioritized over oncosis. When the stimulus intensity is larger, the activity duration time is longer and the cell damage is relatively severe, and oncosis is prioritized over apoptosis. For example, a low dose of cisplatin results in apoptosis whereas a high dose induces necrosis (13). It therefore may be concluded that the ATP levels affect the type of cell death that is triggered (12). Numerous studies suggest that apoptosis is associated with the consumption of ATP that initiates cell death, while oncosis is correlated with a lack of ATP during passive cell death. Mallat and Tedgui (14) reported that when the intracellular ATP level is high, apoptosis is induced, while when the intracellular ATP levels are lacking, oncosis is triggered. ATP is produced by ATP synthase, which is a protein complex located within the mitochondrial membrane that binds ADP and a phosphate group together to produce ATP. ATP6 is a subunit of the F0 complex of transmembrane F-type ATP synthase. ATP8 is a protein that, in humans, is encoded by the MT-ATP8 gene and acts as a functional subunit of mitochondrial ATP synthase. This subunit appears to be an integral component of the stator stalk in mitochondrial ATP synthase, which is composed of spherical F1 and F0 subbases. Mitochondrial ATP synthase, or complex V, generates $>95 \%$ of cellular ATP. Complex V is a multisubunit complex consisting of two functional domains, F1 and F0, connected by a stalk. The F0 domain is embedded in the mitochondrial inner membrane and consists of eight subunits. Two of the F0 subunits, subunit a (or subunit 6) and subunit A6L (or subunit 8) are encoded by the mtDNA ATP6 and ATP8 genes, respectively (15). One study identified that mithochondrial ATP6 expression for maintaining stability had an important role in the mitochondrial genome. A number of studies have suggested that when cells are damaged, cellular stress increases ATP6 gene expression, which has a 
role in stabilizing the mitochondrial genome and protecting cells $(11,16)$. The extent of ATP8 downregulation and ATP6 upregulation is positively correlated with the degree of cellular damage. Regardless of whether ATP6 expression is downregulated or ATP8 is upregulated, ATP synthase becomes dysfunctional, which reduces the subsequent ATP levels, leading to cell oncosis.

CYPD, a mitochondrial peptidyl prolyl cis-trans isomerase that regulates mitochondrial permeability transition, has been demonstrated to control oncosis activated by diverse stimuli (17). CYPD is considered to regulate the opening of the permeability transition pore via its interactions with adenine nucleotide translocator (ANT). These interactions are inhibited by cyclosporine A, which supports the hypothesis that cyclophilin D has a role in pore opening (18). CHMP6 decreased the cell mitochondrial membrane potential and was involved in the regulation of cell oncosis. Cell death induced by CHMP6 was shown to be a caspase-independent mechanism, as anti-apoptotic gene Bcl-xl and caspase family inhibitors were observed to only exhibit a weak effect on the induction of cell death (19). The mitochondrial membrane permeability transition (MPT) is a $\mathrm{Ca}^{2+}$-dependent increase in mitochondrial membrane permeability that leads to mitochondrial swelling, and rupture of the outer mitochondrial membrane. The MPT is hypothesized to occur following opening of the permeability transition pore, which putatively consists of the voltage-dependent anion channel, the ANT, cyclophilin D and other molecule(s) (20). When cells become damaged, the expression of CYPD is enhanced, as it has protective effects against $\mathrm{Ca}^{2+}$-overload and oxidative stress-induced cell death (21). The present study found that following treatment with DAMA and radiation, the CNE-1 cells over-expressed CHMP6 and CYPD, and began to swell and accumulate a large number of vacuoles. In addition, the organelles were observed to swell and the membrane integrity was damaged. Low dose irradiation only marginally enhanced ATP8 expression and downregulated ATP6. By contrast DAMA combined with irradiation treatment was able to markedly enhance the ATP8 levels and weaken the ATP6 expression. The present study suggests that DAMA exhibits radiosensitiozation for human nasopharyngeal carcinoma cells via a novel form of oncosis-like cell death, leading to ATPase synthase disorder and $\mathrm{Ca}^{2+}$ overload, activation of CHMP and CYPD expression, which ultimately results in mitochondrial permeability transition pore opening, membrane potential dissipation, mitochondrial and cytoplasmic swelling, ATP depletion and the early rupture of the plasma membrane.

In conclusion, the mechanism of oncosis may be a complex process involving numerous proteins and multiple genes. Additional investigations are required to clarify the role of this pathway in the pathogenesis and treatment of human NPC.

\section{Acknowledgements}

This study was supported by grants from the National Natural Science Foundation of China (grant nos. 30660047 and 81060270) and the Guangxi Natural Science Foundation (grant no. 0728112). The authors would like to thank the proteomics department the Medical Scientific Research Centre of Guangxi Medical University for their technical support.

\section{References}

1. Jia WH, Huang QH, Liao J, Ye W, Shugart YY, Liu Q, Chen LZ, Li YH, Lin X, Wen FL, et al: Trends in incidence and mortality of nasopharyngeal carcinoma over a 20-25 year period (1978/1983-2002) in Sihui and Cangwu counties in southern China. BMC Cancer 6: 178, 2006.

2. Liu Y, Hou H, Li DR, et al: Enhancement effect of emodin on radiosensitivity of human nasopharyngeal carcinoma transplanted in nude mice. Chinese Pharmaceutical Journal 45: 1331-1334, 2010.

3. Liu Y, Hou H, Li DR, et al: Correlation of enhancement radiosensitization of emodin isolated from Guangxi P. multiflorum Thunb on hypoxic nasopharyngeal cancer cells with expression of DNA repair genes. Chinese Pharmacological Bulletin 25: 348-352, 2009.

4. Liang Y, Hou HX, Li DR, Qin CM, Chen DL and Wu HH: Synthesis of 1,8-dihydroxy-3-acetyl-6-methyl-9,10 anthraquinone and its inhibition effect on ovarian carcinoma cells SKOV3. Chinese Journal of New Drugs 2012: 1038-1041, 2012 (In Chinese).

5. Miyoshi N, Watanabe E, Osawa T, et al: ATP depletion alters the mode of cell death induced by benzyl isothiocyanate. Biochim Biophys Acta 1782: 566-573. 2008.

6. Lieberthal W, Menza SA and Levine JS: Graded ATP depletion can cause necrosis or apoptosis of cultured mouse proximal tubular cells. Am J Physiol 274: F315-F327, 1998.

7. Levin S: Apoptosis, necrosis, or oncosis: what is your diagnosis? A report from the Cell Death Nomenclature Committee of the Society of Toxicologic Pathologists. Toxicol Sci 41: 155-156, 1998.

8. Zhou X, Sun WJ, Wang WM, et al: Artesunate inhibits the growth of gastric cancer cells through the mechanism of promoting oncosis both in vitro and in vivo. Anticancer Drugs 24: 920-927, 2013.

9. Ma LS, Jiang CY, Cui M, et al: Fluopsin C induces oncosis of human breast adenocarcinoma cells. Acta Pharmacol Sin 34: 1093-1100, 2013.

10. Sun L, Zhao Y, Yuan H, et al: Solamargine, a steroidal alkaloid glycoside, induces oncosis in human K562 leukemia and squamous cell carcinoma KB cells. Cancer Chemother Pharmacol 67: 813-821, 2011.

11. Chaudhry MA and Omaruddin RA: Mitochondrial gene expression in directly irradiated and nonirradiated bystander cells. Cancer Biother Radiopharm 26: 657-663, 2011.

12. Gu ML Wang YJ, Shi L, Zhang YB and Chu JY: Comparison on mitochondrial ATP6, ATP8 and Cyt b genes between Chinese Tibetans in three different zones: detecting the signature of natural selection on mitochondrial genome. Yi Chuan 31: 147-152, 2009 (In Chinese).

13. Lieberthal W, Triaca V and Levine J: Mechanisms of death induced by cisplatin in proximal tubular epithelial cells: apoptosis vs. necrosis. Am J Physiol 270: F700-F708, 1996.

14. Mallat $Z$ and Tedgui $A$ : Apoptosis in the vasculature: mechanisms and functional importance. Br J Pharmacol 130: 947-962, 2000.

15. Contamine V and Picard M: Maintenance and integrity of the mitochondrial genome: a plethora of nuclear genes in the budding yeast. Microbiol Mol Biol Rev 64: 281-315, 2000.

16. Arnold RS, Sun CQ, Richards JC, et al: Mitochondrial DNA mutation stimulates prostate cancer growth in bone stromal environment. Prostate 69: 1-11, 2009.

17. Nakagawa T, Shimizu S, Watanabe T, et al: Cyclophilin D-dependent mitochondrial permeability transition regulates some necrotic but not apoptotic cell death. Nature 434: 652-658, 2005.

18. Baines CP, Kaiser RA, Purcell NH, et al: Loss of cyclophilin D reveals a critical role for mitochondrial permeability transition in cell death. Nature 434: 658-662, 2005.

19. Fu D, Tian L, Peng Z, et al: Overexpression of CHMP6 induces cellular oncosis and apoptosis in HeLa cells. Biosci Biotechnol Biochem 73: 494-501, 2009.

20. Tsujimoto Y and Shimizu S: Role of the mitochondrial membrane permeability transition in cell death. Apoptosis 12: 835-840, 2007.

21. Javadov S and Kuznetsov A: Mitochondrial permeability transition and cell death: the role of cyclophilin d. Front Physiol 4: 76, 2013. 